\title{
EARLY PUSHING URGE IN LABOUR AND MIDWIFERY PRACTICE: A PROSPECTIVE OBSERVATIONAL STUDY AT AN ITALIAN MATERNITY HOSPITAL
}

\author{
Sara E. Borrelli, Anna Locatelli, Antonella Nespoli
}

\begin{abstract}
Objective. To investigate the early pushing urge (EPU) incidence in one maternity unit and explore how it is managed by midwives. The relation to some obstetric outcomes was also observed but not analysed in depth. Design. Prospective observational study. Setting. Italian maternity hospital. Sample. 60 women (44 nullips and 16 multips) experiencing EPU during labour. Findings. The total EPU incidence percentage was $7.6 \%$. The single midwives' incidences range had a very wide margin, noting an inverse proportion between the number of diagnoses of EPU and midwife's waiting time between urge to push and vaginal examination. Two care policies were adopted in relation to the phenomenon: stop pushing technique $(\mathrm{n}=52 / 60)$ and "let the woman do what she feels" technique $(n=8 / 60)$. In case of stop pushing techniques, midwives proposed several combined techniques (change of maternal position, blowing breath, vocalization, use of the bath). The EPU diagnosis at less than $8 \mathrm{~cm}$ of cervical dilatation was associated with more medical interventions. Maternal and neonatal outcomes were within the range of normal physiology. An association between the dilatation at EPU diagnosis and obstetric outcomes was observed, in particular the modality of delivery and perineal outcomes.
\end{abstract}

Conclusions and implication for practice. This paper contributes new knowledge to the body of literature around the EPU phenomenon during labour and midwifery practices adopted in response to it. Overall, it could be argued that EPU is a physiologic variation in labour if maternal and foetal conditions are good. Midwives might suggest techniques to woman to help her to stay with the pain, such as change of position, blowing breath, vocalization and use of the bath. However, the impact of policies, guidelines and culture on midwifery practices of the specific setting are a limitation of the study because it is not representative of other similar maternity units. Thus, a larger scale work should be considered, including different units and settings. The optimal response to the phenomenon should be studied, considering EPU at different dilatation ranges. Future investigations could also focus on qualitative analysis of women and midwives' personal experience in relation to the phenomenon. Key words: Early Pushing Urge, labour, second stage, midwifery practice. 


\section{INTRODUCTION}

The nature of physiological childbirth is highly debated. The results of research during past decades have contributed to clinicians' reconsideration of the definition of second stage of labour. The traditional definition states that it starts with full dilatation of cervix and finishes with birth (Roberts, 2004). However, some women may experience the urge to push before the full dilatation of cervix: this phenomenon is clinically called early pushing urge (EPU). The diagnosis of EPU is made with the perception of irresistible urge to push by the woman before full cervical dilatation, confirmed by vaginal examination (Downe, 2008).

A series of questions about the phenomenon arose: how frequently does the phenomenon of early pushing urge occur? How do midwives manage EPU? It could be debated that early pushing urge is an unpredictable and non-preventable phenomenon that cannot be avoided through specific clinical management strategies. Indeed, the midwife can often act only after the appearance of the phenomenon. There is no scientific evidence on the optimal response to the EPU (Perez, 2006).

The aim of this study was to investigate the early pushing urge (EPU) incidence in one delivery unit and explore how it is managed by midwives. The relation to some obstetric outcomes was also observed but not analysed in depth.

\section{BACKGROUND}

The nature of the physiology of the second stage labour has been subject of debate for many years (Buxton \& Redman, 1990; Cohen, 1977; Downe, 2003; Maresh, 1987; McCandlish et al., 1998; Petersen \& Besuner, 1997; Roberts et al., 1987; Roberts \& Woolley, 1996; Roberts, 2002; Roberts et al., 2004; Roberts \& Hanson, 2007; Sampselle et al., 2005; Simkin, 1986; Yeates \& Roberts, 1984).

While most scientific research was conducted in the area of organised versus spontaneous pushing efforts, or delayed compared to the immediate pushing following the 
diagnosis of full dilatation of the cervical os in the context of epidural analgesia, the topic of early pushing urge appears to be less discussed (Roberts, 2004). There are controversies about the prevalence of EPU, the nature of the phenomenon and the optimum approach to management (Perez, 2006).

Berkley (1931) affirmed that no good can be done by bearing down before the dilatation of the cervical os is complete or nearly complete. Other authors, although they appear to be strong supporters of physiology of labour, agree, stating that pushing before complete dilation of the cervix can be harmful. Benyon (1957) states categorically that everyone now accepts that pushing before full dilatation is both useless and harmful and condemns it utterly. Gaskin (1990) makes a brief reference to the technique of avoiding the push if the dilatation is not complete, waiting further time. Gaskin says that pushing before complete dilation can increase the risk of cervical trauma and the onset of uterine prolapse, suggesting that the practitioner should reduce the lip of cervix, if it is present. Therefore, it has been traditionally assumed that the early urge to push is pathological, and that it will lead to maternal exhaustion and/or cervical oedema or trauma.

More recent researchers (Enkin, 2000; Petersen \& Besuner, 1997; Roberts, 1987) indicate that EPU may be experienced by a significant minority of women, suggesting that, in certain circumstances, it may be physiological. Until recently, both researchers and obstetric textbooks have generally presented EPU as a phenomenon that can be avoided through specific practices such as changing maternal posture and techniques of breathing (Davis-Floyd, 1997). However, it is still not clear whether these findings are influenced by factors such as maternal posture, foetal position or parity, and there is not enough evidence to determine the optimal response to the phenomenon. Recent editions of obstetric textbooks suggest that the evidence on the impact of EPU on maternal and fetal outcomes is equivocal and there is no evidence on the optimal response to the phenomenon (Downe, 2003). Downe (2003) claims that the midwife needs to work with 
each individual woman in the context of each labour to determine the best approach in the specific case.

The earliest research in this area appears to be Yeates \& Roberts (1984), who found that nine out of ten women experienced the EPU. Roberts (1987) undertook another research project, with the explicit aim of investigating the phenomenon of early pushing urge. She found that $54 \%$ of women $(n=17 / 31)$ experienced EPU at less than $9 \mathrm{~cm}$ of cervical dilation.

Downe (2008) explored the incidence of diagnosis of EPU by a sample of English midwives finding an incidence of $20 \%$ ( $n=153 / 765$ women in labour) and examined the practice of midwives in relation to the phenomenon. This result appears to be at odds with the previously published literature and Downe (2008) states that the estimate of $20 \%$ women experiencing the EPU is probably artificially low. However, this is still a significant proportion of women and her results provide ample justification for carrying out a study in this area.

Downe (2008) cites an extensive audit carried out in 1994-1995 in USA by the Association of Women's Health, Obstetrics and Neonatal Nurses (AWHONN), involving more than 1500 midwives of 40 maternity units located in USA and Canada. Nearly $93 \%$ of respondents reported that they had witnessed the EPU in labouring women. In case of early pushing urge, $45.5 \%$ of midwives claimed to implement a policy of a non-push technique involving a blowing breath in order to minimize the risk of cervical laceration and oedema. Blowing breath is a slow, shallow breath that is done while the woman is relaxed, not forcing herself to go beyond what is comfortable. Beginning with this cleansing breath, the woman then gently inhales through her nose and, on the exhale, softly blows through her mouth, ending with another cleansing breath (Lothian \& De Vries, 2010). The Guide to Effective Care in Pregnancy and Childbirth (2000) concludes that if the mother feels that she wishes to start pushing when the cervix is less than $8 \mathrm{~cm}$ dilated, the woman should be asked to find the position in which she feels most comfortable and 
try to resist the urge to push by trying alternatives such as breathing techniques. If there is only a rim of cervix left and the woman has an irresistible urge to push, she may feel better doing so as, the authors suggest, it is unlikely that any harm will come from spontaneous pushing before full dilatation providing she does not exhaust herself.

After noting the lack of studies about midwifery practice in relation to the early pushing urge, Downe (2008) undertook two mixed methods projects in UK. Practice implemented by midwives in case of EPU were: change of maternal position, maternal mobilization, breathing techniques, pharmacological and alternative pain relief, manual reduction of the cervix, back massage, use of the bath, aromatherapy, giving information and supporting the labouring mother (midwives that offered support were equally divided between support to stop pushing and to "go with her instinct"). Midwives tended to differ in their approach depending on the parity of the woman and the stage of cervical dilatation (nullips who were pushing at earlier dilatations were actively discouraged from pushing more than multips pushing at later dilatations). Fear of cervical oedema or damage, avoiding maternal and foetal stress and supporting the physiological process of labour were the main drivers for the midwives' responses to the EPU. Observing women in labour was the most powerful influence on midwifery practice. Other influencing factors were: midwifery training, peer influence, teachings of mentors and having own babies. Downe (2008) concludes that the same woman may respond differently depending on the accompanying midwife in childbirth, and how different midwives, while watching the same maternal behaviour, can come to different conclusions about the physiology or pathology of the event.

If EPU does occur so regularly, it may be a physiological process for some women. However, there is no definitive evidence from research studies of the consequences for mother or baby when comparing different support approaches to the phenomenon. The literature review raises interesting questions as there is no agreement about the real incidence of EPU, the nature of the phenomenon and the optimum midwifery practice in 
response to it. There is a clearly need for further research on all aspects of EPU in order to recommend midwifery practices based on sound evidence. This article aims to contribute new knowledge in the body of literature around the EPU phenomenon during labour and midwifery practices adopted in response to it.

\section{METHODS}

A prospective observational approach was undertaken in an Italian maternity hospital with approximately 3000 births/year. Researchers asked the collaboration of midwives working in the delivery unit of the hospital in order to collect data about women and midwifery practice, using a data collection sheet. Twenty-two midwives agreed to contribute to the data collection.

The women's sample included 60 pregnant women who manifested the event (Early Pushing Urge) in labour at term pregnancy, with a single foetus in cephalic presentation. Exclusion criteria were foetal malformations and women under eighteen (due to difficulties procuring informed consent).

The recruitment period was six months (from July $1^{\text {st }}$ to December $31^{\text {st }}, 2009$ ). In the first and second stage of labour, midwifery care followed local protocols, where the midwife is the professional responsible for physiological labour and delivery. The gynaecologist would intervene in case of pathology or doubt in diagnosis. If possible, the same midwife is present in the delivery room with the woman during the entire period of labour and birth, assuring continuity of care, one-to-one individualised care and privacy. The working shifts of twelve hours were a facilitator for this. In case of physiological labour, vaginal examination is done every two hours by the midwife, and is recorded on the partogram. There is not a dedicated section in the woman's case history to record the appearance of EPU. There are no hospital guidelines or protocols to which midwives could refer in the case of EPU. As requested by researchers, the midwife documented EPU appearance during labour and advice given to woman on the provided data collection sheet. The 
diagnosis of EPU was made with the perception of irresistible urge to push by the woman before full cervical dilatation, confirmed by vaginal examination. Midwives collected demographic data, and maternal and foetal/neonatal parameters about the labour and birth.

The study protocol was approved by the local Ethics Committee. Women and midwives' written consents were required prior to participation and anonymity of participants was guaranteed.

\section{DATA ANALYSIS}

All variables considered in the data collection sheet were registered in Epi-Info 3.5.1 statistical software. A descriptive analysis was performed using Epi-Info 3.5.1 data analysis. The interdependence between variables, the possible correlation between characteristics of EPU and care interventions were then investigated.

\section{FINDINGS}

Sample population. The characteristics of the sample of 60 women are reported in Table 1. The average age was 30.8 years with a range from 20 to 42 years. The gestational weeks at delivery average was 39.6. Enrolled women were for the most part nulliparous $(n=44 / 60)$. Considering women's actual obstetric history, $70 \%(n=42 / 60)$ of pregnancies were physiological. Maternal pathologies included: five cases of polydramnios (8\%), three cases of pre-caesarean section (5\%), three cases of gestational diabetes $(5 \%)$, one case of obesity (2\%), one case of olygohydramnios (2\%) and one case of cholestasis $(2 \%)$. The sample included one case of foetal macrosomy (2\%). Thirteen women (22\%) with a prelabour rupture of membranes were included in the sample. Among these women, 54\% $(n=7 / 13)$ went into spontaneous labour, $23 \%(n=3 / 13)$ were induced and $23 \%(n=3 / 13)$ augmented. Among the totality of cases, labours were spontaneous in a rate of $65 \%$ 
$(n=39 / 60)$. The percentage of inductions was $17 \%(n=10 / 60)$ and labours were augmented in the $18 \%$ of cases $(n=11 / 60)$.

\begin{tabular}{lccc}
\hline Characteristics of the sample & $\boldsymbol{\mu}$ & Min. value & Max. Value \\
\hline Age & 30.8 & 20 & 42 \\
Maternal weight & $73 \mathrm{Kg}$ & $52 \mathrm{Kg}$ & $93 \mathrm{Kg}$ \\
Gestational Weeks at delivery & 39.6 & 37.1 & 43 \\
LSF & $34.8 \mathrm{~cm}$ & $30 \mathrm{~cm}$ & $40 \mathrm{~cm}$ \\
\hline & $\mathbf{n}$ & $\%$ & \\
\hline Nullips & 44 & 73 & \\
Multips & 16 & 27 & \\
\hline Primigravid & 35 & 58 & \\
Multigravid & 25 & 42 & \\
\hline Physiological pregnancy & 42 & 70 & \\
Pathological pregnancy & 18 & 30 & \\
\hline PROM & 13 & 22 & \\
Spontaneous labour & 39 & 17 & \\
Induced labour & 10 & 55 & \\
Accelerated labour & 11 & & \\
\hline
\end{tabular}

Table 1. Characteristics of the sample

Incidence of Early Pushing Urge in labour. At the end of the enrolment period, the midwives recorded 60 cases of EPU on a total number of 789 births. The total EPU incidence percentage was $7.6 \%(n=60 / 789)$ and the average of incidence per midwife was $7.8 \%$. The two rates correspond, supporting data's validity. The single midwives' 
incidences range has a very wide margin, from a minimum value of $2.3 \%(n=1 / 44)$ to a maximum value of $20 \%(n=4 / 20)$.

The number of diagnoses of EPU proportionally decreases the longer midwives wait to investigate it. Sixty percent $(n=36 / 60)$ of EPU were diagnosed within a 30 minute period between the behaviour manifesting and the midwife undertaking a vaginal examination: only 4 cases $(7 \%)$ were identified if the midwife waited over 90 minutes.

The diagnosis of EPU appears to be related to parity, as it was experienced by 44 nulliparous women (73\%) and 16 multiparous women (27\%). EPU was associated with a foetus in posterior position in $41 \%(n=25 / 60)$ of cases and with deflected head in $30 \%$ ( $n=18 / 60)$ of cases. In 16 cases $(27 \%)$ midwives found both present. Most of these cases were identified between 3 and $7 \mathrm{~cm}$ of dilatation. It may be that both conditions predisposed to EPU.

Early Pushing Urge in labour and midwifery practice. Forty-three percent $(n=26 / 60)$ of EPU were diagnosed within the range of $8-9 \mathrm{~cm}$ of cervical dilatation, $32 \%(n=19 / 60)$ at 6 $7 \mathrm{~cm}$ of dilatation and $23 \%(n=14 / 60)$ at $4-5 \mathrm{~cm}$ of dilatation. EPU was diagnosed at $3 \mathrm{~cm}$ of dilatation only in one case $(2 \%)$. Occurrences at each $\mathrm{cm}$ of dilatation are reported in Table 2 


\begin{tabular}{ccc}
\hline $\begin{array}{c}\text { Dilatation at EPU } \\
\text { diagnosis }\end{array}$ & N women & $\%$ \\
\hline 9 & 13 & 22 \\
\hline 8 & 13 & 22 \\
\hline 7 & 9 & 15 \\
\hline 6 & 10 & 16 \\
\hline 5 & 9 & 15 \\
\hline 4 & 5 & 8 \\
\hline 3 & 1 & 2 \\
\hline TOTAL & 60 & 100 \\
\hline
\end{tabular}

Table 2. Dilatation at EPU diagnosis

The next research objective was to investigate midwifery practice in response to EPU. There were two approaches to the phenomenon: stop pushing technique in $87 \%$ of cases $(n=52 / 60)$ and "let the woman do what she feels" technique in $13 \%$ of women $(n=8 / 60)$. With the first approach midwives recommended number of techniques to women, including change of maternal position in 45 women (75\%), blowing breath in 29 cases (48\%), vocalization in 16 women (27\%) and the use of a bath in 9 cases (15\%). Change of position combined with blowing breath was $30 \%(n=18 / 60)$ of cases and change of position combined with vocalization was used in $15 \%(n=9 / 60)$ of women. Analysing in detail types of maternal position's change, it was found that the most frequent was the hands and knees posture with a rate of $78 \%(n=35 / 60)$ followed by lateral position $9 \%$ $(n=4 / 60)$ of the time. The combination of both was used in $13 \%(n=9 / 60)$ of cases.

Considering the cases in which the midwife proposed the use of a bath, it occurred more frequently in women with a dilatation of $4-5 \mathrm{~cm}$ at EPU diagnosis, with a rate of $36 \%$ $(n=5 / 14)$. The dilatation at EPU diagnosis seems not to influence the other midwifery practices (change of maternal position, blowing breath and vocalization). 
Some midwives recommended a medical intervention after the appearance of the phenomenon: epidural analgesia in 9 cases (15\%), reduction of anterior cervical lip in 6 women (10\%), amniotomy in 2 cases (3\%) and oxytocin infusion only in 1 woman (2\%). The frequency and the choice of medical approaches seemed to be related to the dilatation at EPU diagnosis. In fact, epidural analgesia, amniorexi and oxytocic infusion were only employed when EPU was diagnosed before $8 \mathrm{~cm}$ of dilatation with a rate of $20 \%(n=12 / 60)$. Instead, the reduction of anterior cervical lip was used only in the range between $8 \mathrm{~cm}$ and $9 \mathrm{~cm}$ of dilatation, with a total rate of $10 \%(n=6 / 60)$. The EPU diagnosis at less than $8 \mathrm{~cm}$ of cervical dilatation was associated with more medical interventions. However, it is not clear whether medical interventions made any difference to the outcomes.

Maternal and foetal/neonatal outcomes. Maternal and neonatal outcomes were very good with $93 \%(n=56 / 60)$ spontaneous delivery rate, $3 \%(n=2 / 60)$ vacuum extractor, $2 \%$ $(n=1 / 60)$ caesarean section and $2 \%(n=1 / 60)$ Kristeller manoeuvre (pression on the utherine fundal synchronously with the uterine contraction in the second stage of labour). Thirty-nine percent $(n=23 / 60)$ of women had an intact perineum and there were no third degree tears, cervical laceration or postpartum haemorrhages. Neonatal outcomes were also excellent.

An association between the dilatation at EPU diagnosis and some maternal outcomes has been observed. All operative deliveries (vacuum extractor, caesarean section and Kristeller manoeuvre) occurred when EPU was diagnosed at 5-6 cm of dilatation. All first degree tears were observed only among the cases in which EPU was diagnosed at 8-9 $\mathrm{cm}$ of dilatation, with a total rate of $12 \%(\mathrm{n}=7 / 60)$. Furthermore, a higher rate of episiotomies occurred between 5 and $7 \mathrm{~cm}$ of dilatation at EPU diagnosis, with a percentage of $35 \%(n=10 / 28)$. However, these associations need further investigation as 
due to the small scale of the study it is impossible to determine if there is a real correlation or an incidental finding.

\section{DISCUSSION}

This is the first study that investigates the phenomenon of EPU in an Italian maternity setting. However, it must be acknowledged that this is a small study in a local context. The policies and guidelines implemented by this hospital pay attention to the autonomy and responsibility of the midwife as explained in the method section. Furthermore, the greatest part of midwives seems to respect the normalcy of labour and birth. For all these reasons, the setting and the midwifery practices could not be representative of those of the other delivery units. However, there are some findings that will be of interest and benefit to midwifery practice.

Considering the body of literature, the EPU incidence rate is much lower than expected, if compared with Roberts (1987) and Downe (2008) results. In fact, Roberts (1987) found that $54 \%$ of women experienced EPU at less than $9 \mathrm{~cm}$ of dilation and Downe (2008) observed a total incidence of EPU of $20 \%$. The authors are aware that the study of Downe (2008) has been made on a larger scale and for this reason it might be more reliable and thus representative of the real incidence.

The following question arises from reviewing the findings: could the discrepancy between midwives be explained by hospital policy or variation in diagnosis by midwives? It is more likely to be the latter as the reported incidence varies so much between midwives. If the midwife delays investigating when the woman feels the urge to push, she is more likely to find the cervix completely dilated at the vaginal examination and therefore less likely to diagnose an EPU. In fact, the number of diagnoses of EPU proportionally decreases the longer midwives wait to investigate it. If an early diagnosis could facilitate the response of the midwife to the phenomenon, the question that arises is whether missing some EPU diagnosis might decrease unnecessary medical interventions. 
In the past, the dominant idea about EPU was mainly related to the pathology of the event and to the potential harm in bearing down before the full cervical dilatation (Berkley, 1931; Benyon, 1957; Gaskin, 1990). The concept of EPU as physiological event if occurring within good maternal and foetal conditions is relatively recent (Downe, 2003). The results of this study match with this last position, as maternal and neonatal outcomes were very good when EPU occurred. Following the philosophy of individualised and woman-centred care supported by Downe (2003), when midwives had diagnosed EPU and requested women not push they advised different techniques in relation to each particular situation, giving the woman the possibility of choice. In some cases, a combination of different techniques was used, indicating again the opportunity for the woman of finding herself "what worked" for her body.

Taking into consideration maternal position change, midwives mostly advised women to adopt a hands and knees posture, lateral position or a combination of both. It is of interest that they have chosen these two positions as there are no hospital policies or guidelines referring to the optimum practice in relation to EPU. Furthermore, there are no guidelines or recommendations in relation to EPU published by the National Institute for Health and Clinical Excellence UK nor by the Italian Society of Gynaecology and Obstetrics.

Regarding the frequency and the choice of medical approaches, it is interesting to note how midwives avoid in more than half of 9 -cm-dilatation's cases $(n=9 / 13)$ the suggestion of Gaskin (1990) of reducing the lip of cervix. As an alternative, midwives seem to refer to the philosophy embraced by the Guide to Effective Care in Pregnancy and Childbirth (2000), recommending that the woman should resist the urge to push by choosing the most comfortable position or trying other alternatives such as breathing techniques.

Finally, even if EPU can be considered a physiologic variation in labour if maternal and foetal conditions are good, an association between the dilatation at EPU diagnosis and some obstetric outcomes has been observed, in particular the modality of delivery and perineal outcomes. However, these correlations need further investigation. 


\section{CONCLUSIONS AND IMPLICATION FOR PRACTICE}

The literature review raises interesting questions as there is no agreement about the real incidence of EPU, the nature of the phenomenon and the optimum midwifery practice in response to it. This article aims to contribute new knowledge to the body of literature around the EPU phenomenon during labour and midwifery practices adopted in response to it. Considering the body of literature, the EPU incidence rate in this study is much lower than expected. Our results challenge earlier research about the incidence of EPU though this appears to be mediated by individual midwives threshold for diagnosing it, in particular, how long they wait between observing the EPU and doing a vaginal examination to see if the woman's cervix is fully dilated.

The results suggest that midwives might advise techniques to woman to help her stay with the pain, such as change of position, blowing breath, vocalization and use of the bath. Overall, it could be argued that EPU is a physiologic variation in labour if maternal and foetal conditions are good. However, an association between the dilatation at EPU diagnosis and obstetric outcomes has been observed, in particular modality of delivery and perineal outcomes. These correlations are not clear and need further investigation.

The impact of policies, guidelines and culture on midwifery practices of the specific setting could be considered a limitation of the study because it could not be representative of other similar maternity units. Thus, a larger scale work should be considered, including different units and settings.

The optimal response to the phenomenon should be studied, considering EPU at different dilatation ranges. Further research could explore the link between early bear down up to 7 $\mathrm{cm}$ dilatation and obstetric and neonatal outcomes. The comparison between the two most advised positions (hand and knees and lateral postures) by midwives in case of EPU should be explored. Future investigations could also focus on the qualitative analysis of women and midwives' personal experience in relation to the phenomenon. 


\section{REFERENCES}

Benyon C. (1957) The normal second stage of labour: a plea for reform in its conduct. In:

Kitzinger S., Simkin P. Episiotomy and the second stage of labour. (2nd ed.) Seattle: Pennypress.

Berkeley C., Fairbairn J.S. (1931) White C 1931 Midwifery. (4th ed.) London: Arnold.

Buxton J., Redman C. (1990). Effect of epidural analgesia and delay pushing in the second stage on fetal condition. Contemporary Reviews in Obstetrics and Gynaecology, 2:80-86.

Cohen W.R. (1977) Influence of the duration of second stage labor on perinatal outcome and puerperal morbidity. Obstetrics and Gynaecology, 49: 266-269

Davis-Floyd R.E., Sargent C.F. (1997) Childbirth and authoritative knowledge: crosscultural perspectives. Berkeley: University of California Press.

Downe S. (2003) Transition and the second stage of labour. In: Fraser D., Cooper M. Myles textbook for midwives. (14th ed.) London: Harcourt Health Sciences, 28:509-530.

Downe S. (2008) The early pushing urge: practice and discourse. In: Downe S. Normal Childbirth. Evidence and Debate, 8: 129-148.

Enkin M., Keirse M.J.N.C., Neilson J. et al (2000) A guide to effective care in pregnancy and childbirth. (3rd ed). Oxford: Oxford University Press. 
Gaskin I.M. (1990) Spiritual Midwifery. (3rd ed.) Summertown: The Book Publisher Co.

Jordan B. (1993) Birth in four cultures: a cross-cultural investigation of childbirth in Yucatan, Holland, Sweden and the United States. Champaign: Waveland Press.

Lothian J., De Vries C. (2010) The Official Lamaze Guide: Giving Birth with Confidence. Meadowbrook Press.

Maresh M. (1987) Management of the second stage of labour. Midwife Health Visit Community Nurse, 23:502-504.

McCandlish R., Bowler U., van Asten H., Berridge G., Winter C., Sames L. (1998) A randomized controlled trial of care of the perineum during second stage of normal labour. British Journal of Obstetrics \& Gynaecology, 105:1262-1272.

Perez Botella M., Downe S. (2006) Stories as evidence: the premature urge to push. British Journal of Midwifery, 14 (11): 636-640.

Petersen L., Besuner P. (1997) Pushing technique during labor: issues and controversies. Journal of Obstetric Gynaecology \& Neonatal Nursing, 26:719-726.

Roberts J.E., Goldstein S.A., Gruener J.S. et al (1987) A descriptive analysis of involuntary bearing-down efforts during the expulsive phase of labor. Journal of Obstetric Gynaecology \& Neonatal Nursing, 16:48-55.

Roberts J., Woolley D. (1996) A second look at the second stage of labor. Journal of Obstetric Gynaecology \& Neonatal Nursing, 25(5):415-423. 
Roberts J. (2002) The push for evidence: management of the second stage. Journal of Midwifery \& Womens Health, 47:2-15.

Roberts C.L., Torvaldsen S., Cameron C.A. et al (2004) Delayed versus early pushing in women with epidural analgesia : a systematic review and meta-analysis. British Journal of Obstetric \& Gynaecology, 111:1333-1340.

Roberts J., Hanson L. (2007) Best practices in second stage labor care: maternal bearing down and positioning. Journal of Midifery \& Womens Health, 52:238-245.

Sampselle C.M., Miller J.M., Luecha Y., Fisher K., Rosten L. (2005) Provider support of spontaneous pushing during the second stage of labor. Journal of Obstetric Gynaecology \& Neonatal Nursing, 34:695-702.

Simkin P. (1986) Active and physiologic management of second stage: a review and hypotesis. In: S. Kitzinger, Simkin P. Episiotomy and the second stage of labour. Seattle: Pennypress, 7-21.

Yeates D.A., Roberts J.E. (1984) A comparison of two bearing down techniques during the second stage of labor. Journal of Nursing \& Midwifery, 29:3-11. 